\title{
Primary Collapsing Glomerulopathy
}

National Cancer Institute

\section{Source}

National Cancer Institute. Primary Collapsing Glomerulopathy. NCI Thesaurus. Code C123113.

Collapsing glomerulopathy for which no underlying cause has been identified. 